\title{
The Relationships Between Biological Activities and Structure of Flavan-3-Ols
}

\author{
Cornelia Braicu ${ }^{1}$, Valentina Pilecki ${ }^{2}$, Ovidiu Balacescu ${ }^{1}$, Alexandru Irimie ${ }^{3,4}$ and \\ Ioana Berindan Neagoe ${ }^{1,5, *}$
}

1 Department of Functional Genomics and Experimental Pathology, Cancer Institute "Ion Chiricuta", 34-35 Republicii, Cluj-Napoca 400015, Romania; E-Mails: cornelia.braicu@iocn.ro (C.B.); ovidiubalacescu@iocn.ro(O.B.)

2 Faculty of Biology and Geology, Babes-Bolyai University, 44 Gheorghe Bilascu, Cluj-Napoca 4000006, Romania; E-Mail: valentinapilecki@yahoo.com

3 Department of Surgical Oncology, University of Medicine and Pharmacy, "Iuliu Hatieganu", 8 Victor Babes, Cluj-Napoca 400427, Romania; E-Mail: airimie@umfcluj.ro

4 Department of Surgery, Cancer Institute "Ion Chiricuta”, 34-35 Republicii, Cluj-Napoca 400015, Romania

5 Department of Immunology, University of Medicine and Pharmacy "Iuliu Hatieganu", 8 Victor Babes, Cluj-Napoca 400427, Romania

* Author to whom correspondence should be addressed; E-Mail: ioanaberi@iocn.ro; Tel.: +40-264-450749; Fax: +40-264-598885.

Received: 27 October 2011; in revised form: 23 November 2011 / Accepted: 5 December 2011 / Published: 13 December 2011

\begin{abstract}
Flavan-3-ols are involved in multiple metabolic pathways that induce inhibition of cell proliferation. We studied the structure-activity relationship of gallic acid (GA) and four flavan-3-ols: epigallocatechin gallate (EGCG), epigallocatechin (EGC), catechin (C), and epicatechin (EC). We measured the cell viability by the MTT assay and we determined the concentration of testing compound required to reduce cell viability by $50 \%\left(\mathrm{IC}_{50}\right)$. All tested compounds showed a dose-dependent and time-dependent inhibitory antiproliferative effect on Hs578T cells; $\mathrm{IC}_{50}$ values varying from the 15.81 to $326.8 \mu \mathrm{M}$. Intracellular ROS (reactive oxygen species) were quantified using a fluorescent probe 2',7'-dichlorofluorescin diacetate (DCFH-DA). Only the treatment with $10 \mu \mathrm{M}$ EGC and EGCG was able to induce a significant decrease of ROS concentration and increased levels of ROS were registered for $100 \mu \mathrm{M}$ EGCG, EGC and GA. Flavans-3-ols and GA induced apoptosis in a dose- and
\end{abstract}


time-dependent manner, which indicated that the induction of apoptosis mediated their cytotoxic activity at least partially. The galloylated catechins have shown a stronger antiproliferative activity and apoptotic effect than the one produced by non galloylated catechins. The galloylated flavan-3-ols are potential therapeutic agents for patients with triple negative breast cancer via induction of apoptosis.

Keywords: catechins; structure-activity relationship; gallate moiety; cell proliferation; apoptosis; pro/antioxidant

\section{Introduction}

The class of natural compounds with biological active properties such as polyphenols has attracted attention in terms of beneficial effects on human health, due to their low toxicity, low cost and high availability. Catechins and catechin gallates belong to the class of polyphenol compounds, subclass of flavan-3-ols, which include (-)-epigallocatechin-3-gallate (EGCG), (-)-epigallocatechin (EGC), $(-)$-epicatechin (EC), (+)-catechins (C) and (-)-epicatechin-3-gallate (ECG). EGCG and ECG contain a gallic acid moiety at position 3 on the $\mathrm{C}$ ring (Figure 1) [1-7].

Figure 1. Chemical structure of catechins and gallic acid (GA); the gallate moiety is circled.

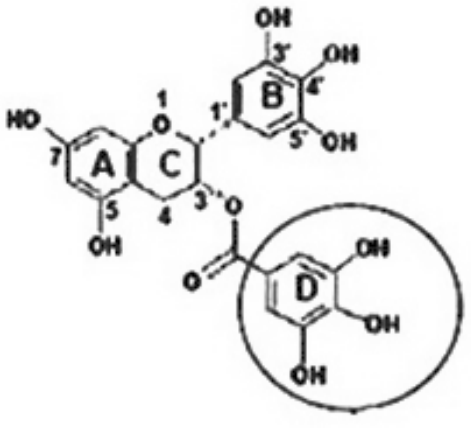

(-)-epigallocatechingallate (EGCG)

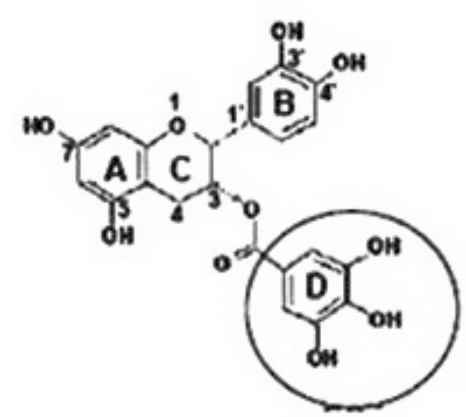

(-)-epicatechin gallate (ECG)

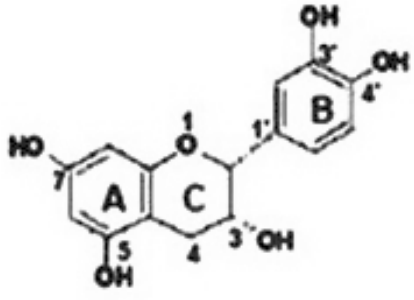

$(-)$-epicatechin (EC)

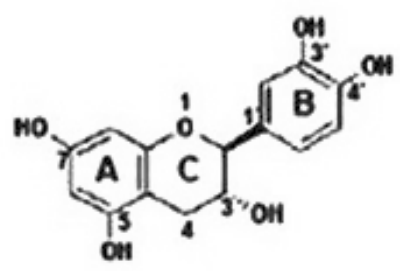

$(+)-\operatorname{catechin}(\mathrm{C})$<smiles>O=C(O)/C=C(/O)CC(O)C(O)O</smiles>

gallic acid (GA)

Flavan-3-ols are characterized by hydroxylated aromatic rings. They are 3-ring phenolic compounds with a double ring attached by a single bond to a third ring and they have multiple hydroxyl groups on the $\mathrm{A}, \mathrm{B}$ and $\mathrm{C}$ rings. The heterocyclic benzopyran ring is known as the $\mathrm{C}$ ring, the fused aromatic ring as the $\mathrm{A}$ ring, and the phenyl constituent as the $\mathrm{B}$ ring. The $\mathrm{A}$ ring can be of two types: a 
phloroglucinol type that is meta-trihydroxylated, or a resorcinol type that is meta-dihydroxylated. The B ring can be monohydroxylated, ortho-dihydroxylated or vicinal-trihydroxylated [1-4]. The catechin and epicatechin are epimers. The structural difference between EGC and EC is an additional hydroxyl group at 5' position of the B ring for EGC. EGCG is an EGC ester derivative, resulting from an esterification at 3 hydroxyl position of the $\mathrm{C}$ ring with a gallate moiety $[2,3]$.

Flavan-3-ols present antioxidant activity in cell-free systems, both in vitro and in vivo. Many mechanisms of action for the flavan-3-ols chemopreventive activities have been proposed [2,4-12]. One of these mechanisms is their pro/antioxidant activity. The antioxidant capacity of flavan-3-ols is proved particularly by their abilities to upregulate antioxidant enzymes and to scavenge the reactive oxygen species (ROS) [2,6].

Flavan-3-ols and their derivatives are a class of phenolic compounds found in green tea leaves, chocolate, grape and grape seeds, having several healthy properties. An anticarcinogenic activity and a protective capacity against oxidative stress-related diseases were previously described $[2,3,10]$. Several flavan-3-ols have been reported as apoptosis inducers and inhibitors of cell proliferation in human tumor cells $[2,4]$. In addition, recent studies indicated their involvement in the modulation of signal transduction pathways, cell survival/death, mitochondrial function and angiogenesis [2-4,6-12].

Most studies have evaluated the biological active properties of EGCG, and only few focused on the relation between the chemical structure and the antiproliferative or anti/prooxidant proprieties of flavan-3-ols, with or without gallate moiety. The development of structure-activity relationships may facilitate the research on cancer therapy. In the present study, we addressed the relation between gallic acid (GA) and flavan-3-ols structures and their activity in Hs579T cell line, an in vitro system for highly invasive triple negative human breast cancer [3]. We determined the cell viability and the concentration of each compound required to reduce cell viability by $50 \%\left(\mathrm{IC}_{50}\right)$. The putative antiapoptotic and anti/prooxidant properties were investigated in order to evaluate a possible relation between structure and activity of flavan-3-ols and GA.

\section{Results and Discussion}

\subsection{The Antiproliferative Effect of Flavan-3-Ols and GA}

At first, we investigated the effect of GA and four types of flavan-3-ols on cultured Hs578T cells. The cells were incubated with the selected compounds, at concentrations between 0-750 $\mu \mathrm{M}$ (Figure 2). The MTT values after 24, 48 and $72 \mathrm{~h}$ incubation are represented as \% of control, in relation with the $\log$ (concentration, $\mu \mathrm{M}$ ) (Figure 2). From these plots, $\mathrm{IC}_{50}$, as well as other statistical parameters, were determined using GraphPad Prism free-trial software (Table 1). The results are presented in Figure 2 and Table 1. A difference of the antiproliferative effect based on the measurement of $\mathrm{IC}_{50}$ was observed in the case of cellular treatment and was ranked as follows: $\mathrm{EGCG}>\mathrm{ECG}>\mathrm{EC}>\mathrm{GA}>\mathrm{C}$ as shows 24 hours' results.

The concentration of GA required to reduce Hs578T cell viability by $50 \%\left(\mathrm{IC}_{50}\right)$ was $211.1 \mu \mathrm{M}$ at $24 \mathrm{~h}, 40.14 \mu \mathrm{M}$ at $48 \mathrm{~h}$, and $31.76 \mu \mathrm{M}$ at $72 \mathrm{~h}$. These results emphasize the fact that gallate moiety is a key component of the antiproliferative effect of tested catechins, by comparing their $\mathrm{IC}_{50}$. The highest 
antiproliferative effect was observed in the case of EGCG, $\mathrm{IC}_{50}$ of $131.6 \mu \mathrm{M}$ at $24 \mathrm{~h}, 15.8 \mu \mathrm{M}$ at $48 \mathrm{~h}$, and $17.75 \mu \mathrm{M}$ at $72 \mathrm{~h}$; $\mathrm{C}$ was less efficient.

Our data showed that all compounds tested exert a dose and time-dependent antiproliferative effect on Hs578T cells. However, we found a differential effect for each compound, with $\mathrm{IC}_{50}$ values varying from the 15.81 to $326.8 \mu \mathrm{M}$. Among the structurally related catechins, the EGCG was the most powerful in inhibiting the growth of breast cancer cells.

Figure 2. The antiproliferative effect as measured by MTT assay after 24, 48 and $72 \mathrm{~h}$, in incubation with different concentrations $(0-750 \mu \mathrm{M})$ of flavan-3-ols or GA on Hs578T cell line; $\log ($ conc, $\mu \mathrm{M})=\log [$ concentration of bioactive compound, $\mu \mathrm{M}]($ mean $\pm \mathrm{SD}, \mathrm{n}=6)$.
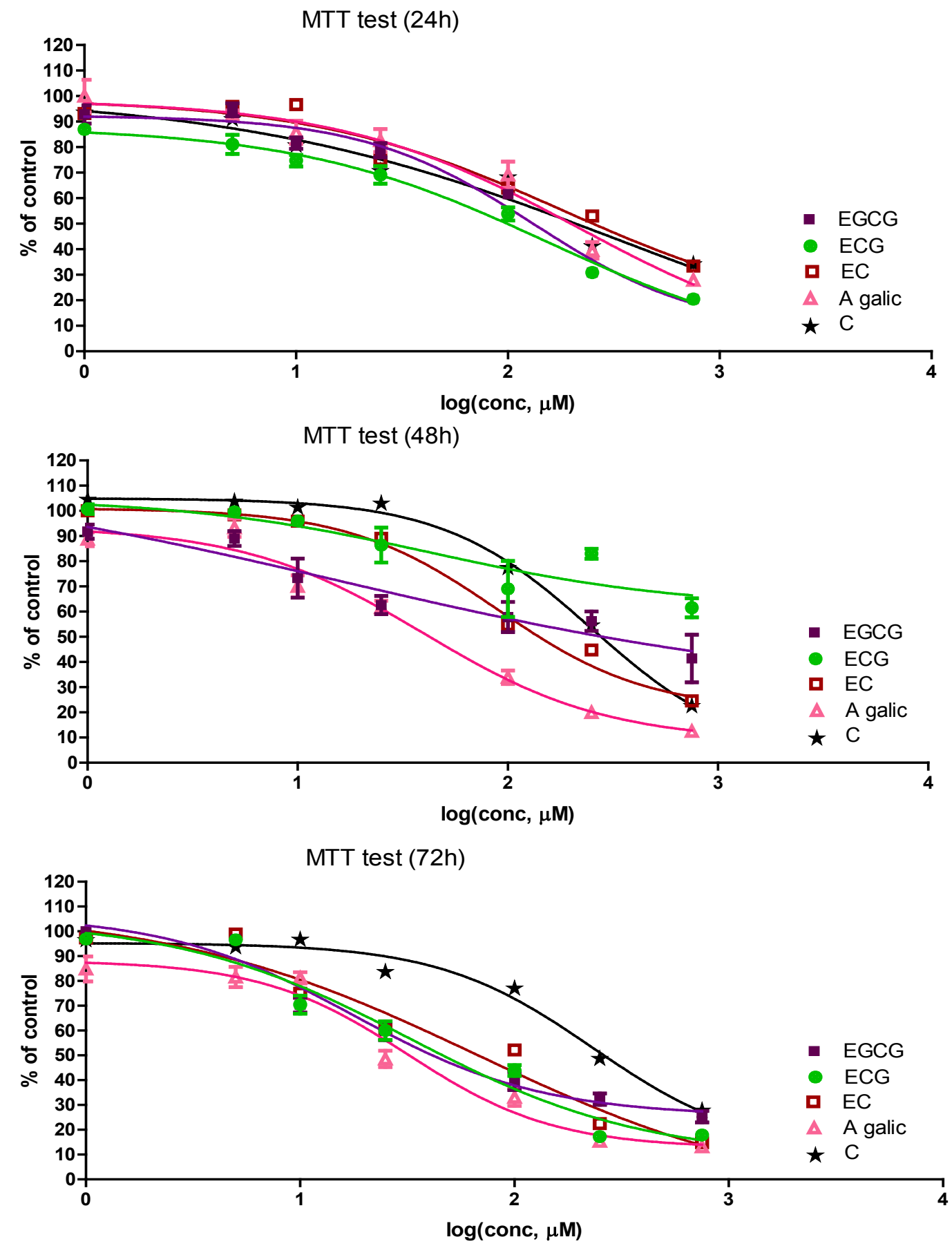
Table 1. $\mathrm{IC}_{50}$ values determined by the MTT test after 24,48 and $72 \mathrm{~h}$ of treatment on Hs578T cell line.

\begin{tabular}{ccccc}
\hline Time (h) & Compound & IC50 $(\boldsymbol{\mu M})$ & Slope & R2 \\
\hline 24 & EGCG & 131.6 & -1.086 & 0.9522 \\
& ECG & 155.1 & -0.7287 & 0.9601 \\
& EC & 180.4 & -0.7303 & 0.7820 \\
& C & 326.8 & -0.4809 & 0.6794 \\
48 & GA & 211.1 & -0.7720 & 0.9016 \\
& EGCG & 15.8 & -0.3760 & 0.6944 \\
& EGC & 43.49 & -0.7227 & 0.5283 \\
& EC & 89.59 & -1.220 & 0.9251 \\
& C & 250.0 & -1.233 & 0.9324 \\
& GA & 40.14 & -1.002 & 0.9424 \\
& EGCG & 17.75 & -0.9903 & 0.9509 \\
& ECG & 32.56 & -0.7985 & 0.9446 \\
& EC & 67.45 & -0.5975 & 0.8716 \\
& C & 233 & -1.205 & 0.9136 \\
& GA & 31.67 & -1.270 & 0.9394 \\
\hline
\end{tabular}

Several studies have evaluated the antiproliferative effect of EGCG, but the other flavan-3-ols with/without gallate moiety received less scientific attention. The present study shows that the gallate moiety is a key component. The EGCG contains two gallate moieties, which could explain the highest antiproliferative effect observed in the present study.

After $48 \mathrm{~h}$ cell treatment with flavan-3-ols and GA, the MTT values obtained were lower than those observed at $24 \mathrm{~h}$ and relatively similar to those registered at $72 \mathrm{~h}$. These results could be explained by their transformation into new metabolic products that induce a higher antiproliferative activity as opposed to the untransformed compound. A similar study [7] demonstrated that some tested phenolic acids exert a dose and time-dependent inhibitory antiproliferative effect on T47D breast cancer cells. Nevertheless, they found a differential effect for each phenolic acid, with $\mathrm{IC}_{50}$ values varying between nanomolar and picomolar range [6]. In our study, the flavan-3-ols show various $\mathrm{IC}_{50}$ values, within the $\mu \mathrm{M}$ range.

Similar differential growth inhibitions were also observed between the human colorectal cancer cell line (Caco-2) and Hs578T, but not in their respective normal counterparts [8]. The proliferation and/or viability of cultured Hs578T and MDA-MB-231 estrogen receptor-negative breast cancer cell lines was proved to be reduced by EGCG treatment in a similar study. Similar negative effects on proliferation were observed with the DMBA-transformed D3-1 cell line. In 1998, Chen et al. evaluated the antiproliferation ability of EGCG in HT-29 cancer cell line. In this study, EGCG inhibited cell proliferation in a dose-dependent manner [6,8]. After $36 \mathrm{~h}$ treatment, EGCG inhibited HT-29 cell growth with an $\mathrm{IC}_{50}$ of approximately $100 \mu \mathrm{M}$ [7]. From Figure 2, it can be observed that the EGCG have the best antiproliferative action at all intervals of time evaluated. The $\mathrm{IC}_{50}$ values for EGCG at $24 \mathrm{~h}$ are in agreement with Chen et al., 1998, but at $48 \mathrm{~h}$ they are much lower.

The difference between EGCG on one side and the C and EGC on the other side is determined by the gallate group; we can suppose that at $24 \mathrm{~h}$ the EGCG treated cells have the best antiproliferative 
activity due to this gallate. The structural differences in flavan-3-ols might explain the differences in the antiproliferative responses. At $48 \mathrm{~h}$, EGCG and other catechins maintained their highest antiproliferative effects with the highest values than those observed at $24 \mathrm{~h}$. Thus, we can assume that after $24 \mathrm{~h}$, the flavan-3-ols were oxidized and the metabolic products were more efficient. This may prove their pro/antioxidant properties [8].

\subsection{Apoptotic Effects of Flavan-3-Ols and GA}

The MTT results showed a reduction in cell proliferation, which might be due to an increase in the apoptotic processes. Thus, we measured the apoptosis rate of Hs578T cells exposed to increasing concentrations of different flavan-3-ols (Figure 3).

Figure 3. Apoptosis evaluation in Hs578T cells after treatment with flavan-3-ols and GA. The cells were stained with Calcein AM and Annexin-Cy5 and were evaluated using the Cell Fluorescence LabChip kit for 2100 bioanalyzer and the results expressed at \% of apoptotic cells at $24 \mathrm{~h}$ and $48 \mathrm{~h}$.

Apoptosis evaluation using Cell Fluorescence LabChip kit for 2100 bioanalyzer

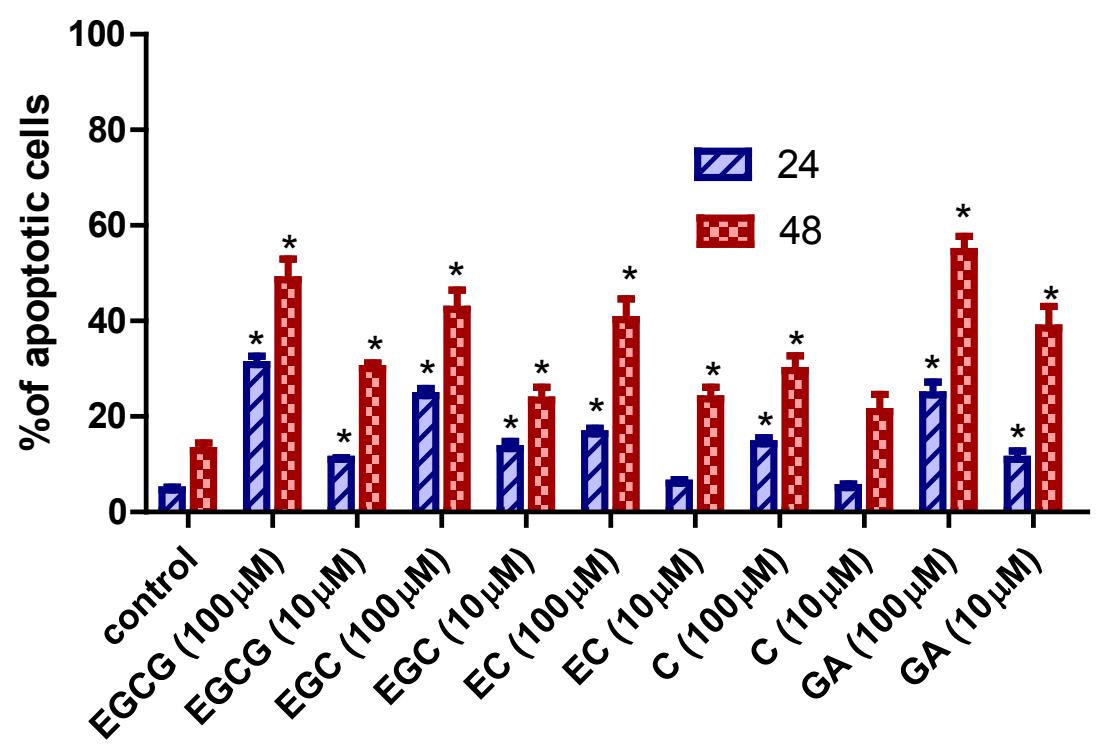

Cell apoptosis was induced by flavan-3-ols treatment as shown by the flow cytometry (Figure 3). Apoptosis may be induced directly by flavan-3-ols or indirectly by their metabolic products [9]. Our data suggest that the hydroxyl groups on the phenolic ring are essential for the antiproliferative and apoptotic activity. The phenolic hydroxyl groups of catechins are primarily responsible for scavenging free radicals, whereas the galloyl moiety is involved in chelating metal ions [10]. The present study revealed a potentially important role of the galloyl structure in the antiproliferative and proapoptotic responses, when comparing the results obtained for GA with those for the flavan-3-ols.

The intrinsic capacity of catechins to form quinone type metabolites upon their oxidation was demonstrated in a recent study [12]. The formation of quinone type metabolites, especially involving the pyrogallol moiety of these catechins [11], can induce the antiproliferative effect, leading to apoptosis. The pyrogallol-type structure plays an important role in the induction of 
apoptosis $[10,13,14]$. These results reveal the fact that flavan-3-ols, such as $\mathrm{C}$ and EC lacking the gallate structure, with only catechol and/or resorcinol groups, are less efficient in inducing apoptosis.

The GA induced apoptotic cell death in human promyelocytic leukemia HL-60 cells. The effect of GA was significantly reduced by blocking ROS [15]. This study suggests the prooxidant action of GA in the induction of apoptosis and confirms our data. On the other hand, the addition of larger alkyl groups to the carboxyl group of GA did not increase the ROS either, but significantly increased its apoptosis-inducing activity. Other studies suggested that the capacity to induce apoptosis involves the extracellular production of ROS [16]. This may be due to the increased lipophilicity of the gallate molecule — a factor that induces apoptosis through a yet unknown mechanism $[14,15,17]$.

\subsection{Detection of Intracellular ROS}

Our unpublished data confirm that the ROS show a steady increase of the fluorescence intensity even in the control group, suggesting that ROS are formed spontaneously in the Hs578T cell line, the maximum fluorescence intensity being registered at $3 \mathrm{~h}$. The effects of two concentrations (10 and $100 \mu \mathrm{M}$ ) of flavan-3-ols and GA at $3 \mathrm{~h}$ after treatment are presented in Figure 4. Only the treatment with $10 \mu \mathrm{M}$ EGC and EGCG was able to induce a significant decrease $(\mathrm{p}<0.05)$ of ROS concentration as compared to the control group and an increased level $(\mathrm{p}<0.05)$ of ROS, as compared to the control group, for $100 \mu \mathrm{M}$ EGCG, EGC and GA.

Figure 4. Effect of flavan-3-ols $(10$ and $100 \mu \mathrm{M})$ at $3 \mathrm{~h}$ after treatment on evaluation reactive oxygen species (ROS) as measured by DCFH-DA fluorescence intensity on Hs578T cells.

\section{Intracellular ROS}

(3 hours after treatment)

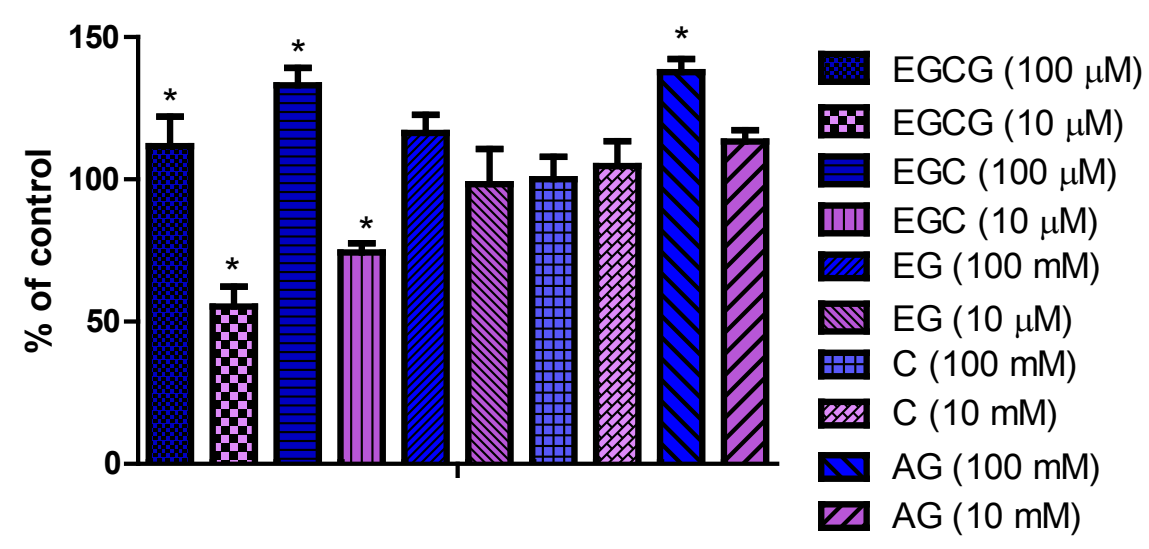

Generally, the health promoting activities of catechins, including the antiproliferative effect, are mainly attributed to their antioxidant capacity and ability to scavenge ROS [18]. The antioxidant effects have been proven on several cellular and molecular targets associated with cell death and cell survival $[2-4,18]$. These properties are due to the presence of the phenolic hydroxy groups on the B ring in ungalloylated catechins (EC and EGC) (Figure 1) and on the B and D rings of the galloylated catechins (ECG and EGCG). The presence of the 3,4,5-trihydroxy B ring has been shown to be 
significant for the antioxidant and radical scavenging activities, and the order of their effectiveness beeing EGCG $>$ EGC $>$ EC $>$ C.

Flavan-3-ols are highly hydrophilic molecules and there are supposed to act as antioxidants in the aqueous compartment [19]. Antioxidant activity of EGCG at physiological concentrations $(0.1-1 \mu \mathrm{M})$ [20], underline the interdependency between the scavenging of radicals in the hydrophilic and hydrophobic environments [21]. Thus, it is suggested that the presence of the gallate group at the 3 position plays the most important role in their free radical-scavenging abilities and an additional insertion of a hydroxyl group at the $5^{\prime}$ position in the $\mathrm{B}$ ring also contributes to their scavenging activities [20,22].

Specific structural components in catechins are reported to be responsible for the different biologic activities, including ROS, as the present study shows [2]. This is confirmed by the present study for all tested compounds at a concentration of $10 \mu \mathrm{M}$. Literature data suggest that the number of hydroxyl groups on the B ring contributes significantly to the ROS scavenging of flavan-3-ols [14]. Recently, the view has changed, and flavonoids are now thought to act as direct antioxidants but rather as inhibitors of prooxidant enzymes or as chelators of transition metals that mask prooxidant actions of reactive nitrogen species (NOS) and ROS [23].

The hydroxyl groups from the three different rings also enhance the inhibition of ROS or induce the prooxidant effect, as it is for EGC and GA. This may be explained by the presence of the ortho-trihydroxyl group in the B ring, which is important for scavenging super-oxide anion, whereas the galloyl moiety is responsible for quenching the hydroxyl radicals [24]. Also, the conjugation between $\mathrm{A}, \mathrm{B}$ and $\mathrm{C}$ rings may provide a resonance effect in the aromatic nucleus that may differentially stabilize the phenoxyl radicals of the different flavan-3-ols structures $[14,25,26]$. The phenoxyl radicals formed during oxidation are quite stable at higher $\mathrm{pH}$ [26].

A large number of studies indicate that tea catechins can act as prooxidants [27,28]. These prooxidant effects are observed under in vitro conditions at pharmacological dose [29]. However, it is still unclear whether the effects exerted on molecular endpoints in signal transduction pathways are downstream events of the modulation of pro/antioxidant balance in cells or they are due to the direct action of EGCG and other catechins on the various molecular targets, independently of the antioxidant activities. Furthermore, most of the putative molecular mechanisms that have been proposed are based on in vitro studies at far higher EGCG concentrations than those achievable in vivo. It has been established that the $\mathrm{B}$ ring, hydroxyl group and galloyl moiety of the catechins are the main contributing factors to their scavenging activities and the presence of the ortho-dihydroxyl group in the B ring and the galloyl moiety are important in maintaining the effectiveness of the radical scavenging ability [29]. Our previous studies have shown that physiologically achievable concentrations of flavan-3-ols protect the cells from ROS induction by mycotoxins [14, 29].

\section{Experimental Section}

\subsection{Cell Culture}

HS578T cells (ECACC) have been grown at $37{ }^{\circ} \mathrm{C}$ in a humidified atmosphere with $5 \% \mathrm{CO}_{2}$ in Dulbecco's Modified Eagle's Medium (DMEM, Sigma-Aldrich, Germany), with 4500 mg/L glucose, 
supplemented with $10 \%$ fetal bovine serum, without sodium pyruvate, $2 \mathrm{mM}$ L-glutamine, $100 \mathrm{U} / \mathrm{mL}$ penicillin and $100 \mathrm{U} / \mathrm{mL}$ streptomycin, $10 \mu \mathrm{g} / \mathrm{mL}$ insuline (Actrapid, Novo Nordisk, Denmark).

\subsection{Cell Treatment}

Cells were treated for 24, 48 and $72 \mathrm{~h}$, with different concentrations $(1,510,25,100,250,750 \mu \mathrm{M})$ of flavan-3-ols (EGCG, ECG, C, EC) or GA, for the evaluation of cell viability by using the MTT test. All reagents were purchased from Sigma-Aldrich. The evaluation of apoptosis was done at 24 and $48 \mathrm{~h}$ after treatment, with 10 and $100 \mu \mathrm{M}$ of the selected compounds.

\subsection{MTT Assay}

$2 \times 10^{4}$ Hs578T cells were treated with flavan-3-ols in a 98-well plate, as described above and incubated for 24, 48 and $72 \mathrm{~h}$, respectively. In order to perform the MTT assay, the culture medium was removed; cells were washed with PBS and $150 \mu \mathrm{L}$ Hanks salt containing MTT (Sigma-Aldrich, Germany). A final concentration of $455 \mu \mathrm{g}$ MTT/mL Hanks salt was added into each well. After 2 hour-incubation under standard conditions, the MTT solution was removed and $200 \mu \mathrm{L}$ of DMSO were added into each well. The absorbance was measured at $490 \mathrm{~nm}$ using a Biotek Synergy HT Microplate Plate Reader.

\subsection{Detection of Intracellular ROS}

Intracellular ROS were quantified by using a fluorescent probe 2'7'- dichlorofluorescin diacetate (DCFH-DA). The DCFH-DA diffuses quickly through the cell membrane and it is enzymatically hydrolyzed by intracellular esterases to non-fluorescent dichlorofluorescin-diacetate (DCFHDA), which is rapidly oxidized to highly fluorescent dichlorofluorescin (DCF) in the presence of intracellular reactive oxygen species. Cells were seeded in 96-well black plates, $2 \times 10^{3}$ cells per well, and allowed to grow for $24 \mathrm{~h}$. After that, the cells were treated with various concentrations of flavan-3-ols and of DCFH-DA (final concentration $0.1 \mu \mathrm{M}$ ). The DCF fluorescence intensity was detected $3 \mathrm{~h}$ after treatment using a Biotek Synergy HT Microplate Plate Reader with $485 \mathrm{~nm}$ emission and $530 \mathrm{~nm}$ excitation wavelengths.

\subsection{On-Chip Flow Cytometry}

The cells were treated as described above and cultured for 24 and $48 \mathrm{~h}$. After incubation, the cells were trypsinized, collected, stained with Anexinn V-biotin Apoptosis Detection kit (Calbiochem) and Calcein AM (Invitrogen) and quantified by on-chip flow-cytometry. The number of apoptotic cells was assessed with Agilent Lab-on-a-chip Bioanalyzer 2100, as percent of apoptotic cells in live cells.

\section{Conclusions}

The antiproliferative and proapoptotic effects of the flavan-3-ols and GA are dose and timedependent. The galloylated catechins showed stronger effects than those of non-galloylated structures. The galloyl moiety appears to be required both for the antiproliferative, apoptotic and antioxidant effects, but there is no clear structure-activity relationship. In conclusion, the results of this study indicate that, 
from all the dietary flavan-3-ols studied, EGCG is the most efficient in the Hs578T breast cancer cell line. EGCG, EGC or GA are able to induce an inhibition of cell proliferation and to modulate apoptosis. Flavan-3-ols may have a dual function, both as antioxidants and pro-oxidants, depending on their concentration and exposure time on the cell culture.

Further studies in this area are needed to determine if catechins or their metabolites are primarily responsible for the health benefits of flavan-3-ols. Moreover, future studies should be based on the investigation of the precise molecular action mechanisms of flavan-3-ols and GA in Hs578T cells. These studies will bring new data on future therapeutic applications and chemopreventive effects in triple negative breast cancer.

\section{Acknowledgments}

This work was partially financed by a grant from the Romanian National University Research Council project PD 533/28.07.2010 "Combining chemotherapeutic effects of flavan-3-ols with RNA interference target therapy in cancer" and partially by a POSCCE 709/2010 grant with title: "Clinical and economical impact of proteom and transcriptom molecular profiling in neoadjuvant therapy of triple negative breast cancer (BREASTIMPACT)".

\section{References}

1. Henning, S.; Choo, J.; Heber, D. Nongallated compared with gallated flavan-3-ols in green and black tea are more bioavailable. J. Nutr. 2008, 138, 1529S-1534S.

2. Velayutham, P.; Babu, A.; Liu, D. Green tea catechins and cardiovascular health: An update. Curr. Med. Chem. 2008, 15, 1840-1850.

3. Thangapazham, R.L.; Passi, N.; Maheshwari, R.K. Green tea polyphenol and epigallocatechin gallate induce apoptosis and inhibit invasion in human breast cancer cells. Cancer Biol. Ther. 2007, 6, 1938-1943.

4. Kampa, M.; Alexaki, V.-I.; Notas, G. Antiproliferative and apoptotic effects of selective phenolic acids on T47D human breast cancer cells: Potential mechanisms of action breast. Cancer Res. 2004, 6, R63-R74.

5. Chen, C.; Shen, G.; Hebbar, V.; Hu, R.; Owuor, E.D.; Kong, A.N. Epigallocatechin-3-gallateinduced stress signals in HT-29 human colon adenocarcinoma cells. Carcinogenesis 2003, 24, 1369-1378.

6. Chen, Z.P.; Schell, J.B.; Ho, C.T.; Chen, K.Y. Green tea epigallocatechin gallate shows a pronounced growth inhibitory effect on cancerous cells but not on their normal counterparts. Cancer Lett. 1998, 129, 173-179.

7. Tang, Y.Q.; Jaganath, I.B.; Sekaran, S.D. Phyllanthus SPP. induces selective growth inhibition of PC-3 and MeWo human cancer cells through modulation of cell cycle and induction of apoptosis. PLoS One 2009; 5, e12644:1-e12644:11.

8. Kavanagh, K.T.; Hafer, L.J.; Kim, D.W.; Mann, K.K.; Sherr, D.H.; Rogers, A.E.; Sonenshein, G.E. Green tea extracts decrease carcinogen-induced mammary tumor burden in rats and rate of breast cancer cell proliferation in culture. J. Cell. Biochem. 2001, 82, 387-398. 
9. Muzolf-Panek, M.; Gliszczyńska-Świglo, A.; de Haan, L.; Aarts, J.M.M.J.G.; Vervoort, J.; Tyrakowska, B.; Rietjens, I.M.C.M. Prooxidant activity of catechins induces EpRE-mediated gene expression. Chem. Res. Toxicol. 2008, 21, 2352-2360.

10. Saeki, K.; Hayakawa, S.; Isemura, M.; Miyase, T. Importance of a pyrogallol-type structure in catechin compounds for apoptosis-inducing activity. Phytochemistry 2000, 53, 391-394.

11. Isemura, M.; Saeki, K.; Kimura, T.; Hayakawa, S.; Minami, T.; Sazuka, M. Tea catechins and related polyphenols as anti-cancer agents. Biofactors 2000, 13, 81-85.

12. Horie, N.; Hirabayashi, N.; Takahashi, Y.; Miyauchi, Y.; Taguchi, H.; Takeishi, K. Synergistic effect of green tea catechins on cell growth and apoptosis induction in gastric carcinoma cells. Biol. Pharm. Bull. 2005, 28, 574-579.

13. Sakagami, H.; Satoh, K.; Hatano, T.; Yoshida, T.; Okuda, T. Possible role of radical intensity and oxidation potential for gallic acid-induced apoptosis. Anticancer Res. 1997, 17, 377-380.

14. Yang, C.S.; Kim, S.; Yang, G.Y.; Lee, M.J. Inhibition of carcinogenesis by tea: Bioavailability of tea polyphenols and mechanisms of actions. Proc. Soc. Exp. Biol. Med. 1999, 220, 213-217.

15. Aldini, G.; Yeum, K.J.; Carini, M.; Krinsky, N.I.; Russell, R. (-)-Epigallocatechin-3-gallate prevents oxidative damage in both the aqueous and lipid compartments of human plasma. Biochem. Biophys. Res. Commun. 2003, 302, 409-414.

16. Chedea, V.S.; Braicu, C.; Socaciu, C. Antioxidant/prooxidant activity of a polyphenolic grape seed extract. Food Chem. 2010, 121, 132-139.

17. Peng, I.-W.; Kuo, S.-M. Flavonoid structure affects the inhibition of lipid peroxidation in Caco-2 intestinal cells at physiological concentrations. J. Nutr. 2003, 133, 2184-2187.

18. Lotito, S.B.; Fraga, C.G. Catechins delay lipid oxidation and alpha-tocopherol and beta-carotene depletion following ascorbate depletion in human plasma. Proc. Soc. Exp. Biol. Med. 2000, 225, $32-38$.

19. Guo, Q.; Zhao, B.; Shen, S.; Hou, J.; Hu, J.; Xin, W. ESR study on the structure-antioxidant activity relationship of tea catechins and their epimers. Biochim. Biophys. Acta 1999, 1427, 13-23

20. Chobot, V.; Huber, C.; Trettenhahn, G.; Hadacek, F. ( \pm )-Catechin: Chemical weapon, antioxidant, or stress regulator. J. Chem. Ecol. 2009, 35, 980-996.

21. Nanjo, F.; Mori, M.; Goto, K.; Hara, Z. Radical scavenging activity of tea catechins and their related compounds. Biosci. Biotechnol. Biochem. 1999, 6, 1621-1623.

22. Heim, K.E.; Tagliaferro, A.R.; Bobilya, D.J. Flavonoid antioxidants: Chemistry, metabolism and structure-activity relationships. J. Nutr. Biochem. 2002, 13, 572-584.

23. Dwibedy, P.; Dey, G.R.; Naik. D.B.; Kishore, K.; Moorthy, P.N. Pulse radiolysis studies on redox reactions of gallic acid: One electron oxidation of gallic acid by gallic acid-OH adductPhys. Chem. Chem. Phys. 1999, 1, 1915-1918.

24. Weisburg, J.H.; Weissman, D.B.; Sedaghat, T.; Babich, H. In vitro cytotoxicity of epigallocatechin gallate and tea extracts to cancerous and normal cells from the human oral cavity. Basic Clin. Pharmacol. Toxicol. 2004, 95, 191-200.

25. Azam, S.; Hadi, N.; Khan, N.U.; Hadi, S.M. Prooxidant property of green tea polyphenols epicatechin and epigallocatechin-3-gallate: Implications for anticancer properties. Toxicol. In Vitro 2004, 18, 555-561. 
26. Azam, S.; Hadi, N.; Khan, N.U.; Hadi, S.M. Prooxidant property of green tea polyphenols epicatechin and epigallocatechin-3-gallate: Implications for anticancer properties. Toxicol. In Vitro 2004, 18, 555-561.

27. Khan, N.; Afaq, F.; Saleem, M.; Ahmad, N.; Mukhtar, H. Targeting multiple signaling pathways by green tea polyphenol (-)-epigallocatechin-3-gallate. Cancer Res. 2006, 66, 2500-2505.

28. Chen, L.-J.; Yang, X.-G.; Shen, S.-R.; Wang, Y.-F. Mechanism of scavenging reactive oxygen radicals of tea catechins. J. Zhejiang Univ. 2002, 28, 563-574.

29. Braicu, C.; Rugina, D.; Chedea, V.S.; Balacescu, L.; Brie, I.; Soritau, O.; Socaciu, C.; Irimie, A. Protective action of different natural flavan-3-ols against aflatoxin B1-related cytotoxicity. J. Food Biochem. 2010, 34, 595-610.

(C) 2011 by the authors; licensee MDPI, Basel, Switzerland. This article is an open access article distributed under the terms and conditions of the Creative Commons Attribution license (http://creativecommons.org/licenses/by/3.0/). 